\title{
Reference Scenarios and Key Performance Indicators for 5G Ultra-dense Networks
}

\author{
Lués Miguel Campos, Luís Ribeiro, \\ Ioannis Karydis, Stelios Karagiannis, \\ Dário Pedro, Jorge Martins and Carlos \\ Marques \\ PDMFC Lisbon, Portugal \\ Email: luis.campos@pdmfc.com
}

\author{
Ana Garcia Armada, Raquel Perez \\ Leal, Manuel José López-Morales \\ Department of Signal Theory and \\ Communications \\ Universidad Carlos III de Madrid \\ 28911 Léganes, Madrid, Spain \\ Email: anagar@ing.uc3m.es
}

\author{
Fernando J. Velez, Pedro Sebastiao, \\ Anderson Rocha Ramos \\ Instituto de Telecomunicações and \\ DEM-Universidade da Beira Interior \\ 6201-001 Covilhã, Portugal \\ Email: fjv@ubi.pt
}

\begin{abstract}
The so-called $5 \mathrm{G}$ will revolutionize the way we live, and work. In order to demonstrate the profound changes, we can expect to experience within the next 5 to 10 years, we present use cases for the planned research within the TeamUp5G project. Some use cases are strongly linked to the network layer and aim at developing solutions capable of optimizing the main promising benefits of 5G: extremely low latency and extremely high bandwidth (e.g., handle video streams, traffic congestion, user profiles), in the most efficient way possible. Other use cases focus on commercial applications that make use of middleware applications to enhance their performance. The latter fall into two main areas: real-time virtual reality and live video streaming, which are extremely demanding in terms of latency and bandwidth to provide an acceptable QoE/QoS to multiple users. The use cases presented are built assuming that $5 \mathrm{G}$ is essential for their support with appropriate QoE/QoS. Key performance indicators and their range of variation are also identified.
\end{abstract}

Keywords-5G, use cases, deployment scenarios, KPIs, characterization parameters

\section{INTRODUCTION}

Over the last two decades, technology has been steadily evolving in a variety of domains: machines are endowed with increasingly powerful processors and larger memory capacities; device-to-device communications open the door to new exquisite inventions and realities; the user population grows more dependent upon technological solutions for everyday problems and needs; quality standards display a never-ending tendency to rise; and the cycle goes on. With $5 \mathrm{G}$ networks promoting larger bandwidth for data transmission and lower latency in communications, potential product and services put on standby due to $4 \mathrm{G}$ limitations are back in the game, along with some innovative ideas.

This paper discusses a series of use cases that explore $5 \mathrm{G}$ features. Despite the promise of $5 \mathrm{G}$ networks lower latencies, it is important to effectively manage massive amounts of data. Consequently, for each use case, it is worthwhile to address issues such as:

- Content distribution based on i) intelligent caching, ii) traffic management by user profile and iii) prefetching.

- Dynamic resource allocation.

This work has received funding from the European Union (EU) Horizon 2020 research and innovation programme under the Marie SkłodowskaCurie ETN TeamUp5G, grant agreement No. 813391, and from the Portguese FCT/MCTES UIDB/EEA/50008/2020 project.
- Intelligent algorithms for optimizing uplink connections.

- Security mechanisms based on network slicing.

On the other hand, the second category of use cases shall develop solutions in the real-time virtual reality (VR) and live Video Streaming (VS) fields. In VR, response times from communication networks are of utmost importance for movement fluidity and to create an authentic sense of reality. It is expected that $5 \mathrm{G}$ networks will allow the diffusion of VR in real-time applications, since larger bandwidth and lower latency may help solve the problem of motion sickness, while at the same time improving video quality in VR devices.

Furthermore, combining live video streaming with recent advancements in Drones and $360^{\circ}$ cameras technology may open a new world of possibilities. Hence, under the latter category, we propose use cases that cover the following general areas: public safety, manufacturing, tourism and entertainment. It is expected that, by involving a variety of industry sectors, the potential of $5 \mathrm{G}$ networks in the development of innovative technologies becomes clearer.

The 5G services and applications include mixed reality (with connectivity and its support on the move [1], [2], [3], [4]), aerial drones as well as opportunities arising from the deployment of $5 \mathrm{G}$ in industry 4.0 [5], [6], [7], while considering resilient authentication and authorization solutions [8], [9], [10], [11].

Based on such vision [1], [12], [13] in this work, we describe a set of structured use cases that will be the basis for the TeamUp5G Innovative Training Network research, and discuss the variation of a set of parameters that will be fundamental in the characterization of these applications, [14].

The remainder of the papers is organized as follows. Section II introduces the TeamUp5G Emergency Drone, Predictive Maintenance in Manufacturing, Jump Travelling, Multiplayer Game Using Virtual Reality (VR), and Live Events using VR/AR uses cases. In Section III the user requirements are analyzed, and the range of variation for the key performance indicators is identified. Section IV draws the conclusions and discusses topics for future research.

\section{USE CASES}

The proposed use cases definition follows a common format including: Context, that explain which scientific field and industry sector the use case is related to; motivation for the need of $5 \mathrm{G}$ networks, or why the use case requires one or 
more of the novel characteristics of 5G networks; high level description of the use case; the initial scenario context; the step-by-step scenario description of the playout of the scenario; and the final scenario.

\section{A. Use case 1: Emergency Drone}

Context - Information and communications are very important in decision-making on emergency situations. When human lives are at risk, the immediately available information may be the difference between life and death. In situations such as earthquakes, fires, floods, etc., it would be very useful to have access to information and maintain communication capabilities, even if all or some of the local infrastructures are not operational. Using drones that can take advantage of the 5G technology, allowing to provide 5G network communications with the decision centers and between crews in the field and even to the population affected by the catastrophe.

Motivation for the need of $\mathbf{5 G}$ networks The $5 \mathrm{G}$ technology will allow data rates 10 or 20 times greater than the $4 \mathrm{G}$ one, better energy efficiency, a much better positioning accuracy, 10 times larger reliability and 100 times more device density. All these features allow for a much better response for Unmanned Aerial Vehicles (UAVs) in emergency situations. For instance, a 4K RAW video streaming at 30 frames per second, consumes about $10 \mathrm{Gbps}$, which is much larger than the peak data rate of $4 \mathrm{G}$. The RAW video from a drone is useful because, for instance, if the drone is flying at a $500 \mathrm{~m}$ altitude, a $45^{\circ}$ photo will cover one square kilometre, meaning that each square meter will roughly be covered by only 8 pixels. This implies that the compression in this case may easily eliminate people in the video. The reduced energy consumption from the $5 \mathrm{G}$ will also be of great value for drone autonomy. With a $5 \mathrm{G}$ micro cell over high population density areas, in case of an event that renders a network failure, a single drone would be able to allow the connection of 1 million devices, one order of magnitude greater than $4 \mathrm{G}$.

Description - The technical and human resources in emergency situations are often limited. Usually in these situations the communications infrastructure is also damaged. The possibility of using UAVs in emergency situations may cover management and communications, if there is any kind of data connection available $(3 \mathrm{G} / 4 \mathrm{G} / 5 \mathrm{G})$. This connection can be provided by other sibling UAV, by creating an array to ensure connectivity. A UAV with capacity to transmit video images and other real time environmental information, as well as assuring the communications between the decision centers and crews and for the general population. There are many possible scenarios where video streams from the disaster area and the reestablishment of connectivity are critical for an adequate response to the emergency. We introduce two very different scenarios as examples:

1 - A large earthquake - A large earthquake over a densely populated metropolitan area would cripple the communications network, especially in the event of a generalized power failure. With the capability of connection one million devices, a few drones with $5 \mathrm{G}$ would be enough to guarantee appropriate coverage of the affected area.

$\mathbf{2}$ - Forest fires - The drone would be able to reach the fire area much before the firefighters, allowing the command center to be aware of the real situation in advance, with the real time video streaming. The $5 \mathrm{G}$ could also allow communication between the crews in the field and the command center which could be hundreds of kilometers away. Danger to villages, roads and other infrastructure would be identified with great advance, allowing the early warning of potential victims and the better organization of the fire response.

Initial scenario - A fire station or emergency station have several UAVs ready to fly on demand. An alert is received by the central authority. The orders for deployment of the nearest UAVs are sent to the local authorities. Also, when the UAV autonomy is near its end, it can return to home or ask where it can be recharged.

\section{Step-by-step scenario}

In both scenarios the first steps are the following:

- UAVs will start travelling to the target area, while being able to receive detailed information about the mission.

- After reaching the target area, UAVs will stream video, thermal and other information to the authorities. Response teams will be able to define priorities.If it is a forest fire event, the drone can autonomously estimate the fire perimeter and identify people/infrastructure in danger.

- UAVs can be independently organized to look for people and identify their presence to the public safety services. They will also be able to recognise connected devices and use triangulation to locate the signal origin by making an allowance for two drones in range. In case of a forest fire event, a drone can also be configured to send SMS and call phones within its reach with an automatic message informing of the danger and asking to call to an emergency number.

- UAVs will be able to create a $5 \mathrm{G}$ micro cell, allowing for connectivity in the affected area. Specifically, in fire events, to target response teams and general population.

Final scenario - The UAV successfully fullfilled the mission and safely returned to its headquarters.

\section{B. Use case 2: Predictive Maintenance in Manufacturing}

Context - With the evolution for beyong 4G communication systems, one of innovations is the adoption of the multi-access edge computing (MEC) architecture through the distribution of datacenters (DCs) on the edge of the network and closer to the end-clients, independently of being residential or enterprise clients. These edge DCs will enable the local deployment of systems based on Big Data and Artificial Intelligence (AI) and analytic applications. By using network slices according to services classes, these DCs will be able to connect to various industrial systems/components. When looking at these types of solutions from the industry perspective, there are several advantages such as: i) Maintenance and operation of network infra-structures demand specific knowledge, thus requiring a considerable investment in an area that is not directly related to their core business, and ii) the new business models based on X-as-aService paradigm enable the optimization of operational costs and reduce the expenditure on infrastructure assets.

Although there are other advantages beside the ones mentioned here, these two are the most visible from the perspective of a closer relationship between the 
telecommunications and manufacturing industries. Augmented Reality (AR) is a good example on how to establish a bridge between the physical and the virtual worlds. AR glasses can be applied in multiple scenarios, allowing the display of digital information in overlay with the real world and the capture of information when used as sensors. Displayed information is customized and adapted to each individual worker, enabling their access to digital tools designed to enhance their performance.

One good example of this practice is the Thyssenkrupp case, which sells and provides maintenance services to elevators and escalators, reducing the average time per assistance by a factor of four. Thyssenkrupp workers use HoloLens glasses to assist them during maintenance jobs, allowing them to be connected to the company's internal operational support systems. Through these glasses, the technicians can locate and identify the malfunctioning components and rapidly repair or replace them. On the most complex cases, the glasses also enable the specialized assistance with bidirectional visual communications: on the one hand the glasses make use of their cameras to capture images and videos and on the other hand they also provide visual assistance in overlay with the real world, using AR.

Motivation for the need of $\mathbf{5 G}$ networks - Besides the Cloud Telco approach associated with 5G, which can be used by verticals to deploy applications and operation support systems, 5G connectivity services will also play a pivotal role. Different service classes map into distinct connectivity requirements that need to be fulfilled to enable the digital transformation of the manufacturing industry. Technologies such as Internet-of-Things (IoT) or AR will only be facilitated by $5 \mathrm{G}$.

Description - Within the context of Industry 4.0, maintenance is one of the areas which attracts more attention and investment due to the foreseen beneficial impact. Herein, the approach has been centered on the use of analytic systems making use of AI to predict the optimal time for maintenance procedures and the use of AR to assist and enhance the performance of technicians. Figure 1 shows a scenario where a manufacturing company uses a platform to assist and optimize the work of their personnel.

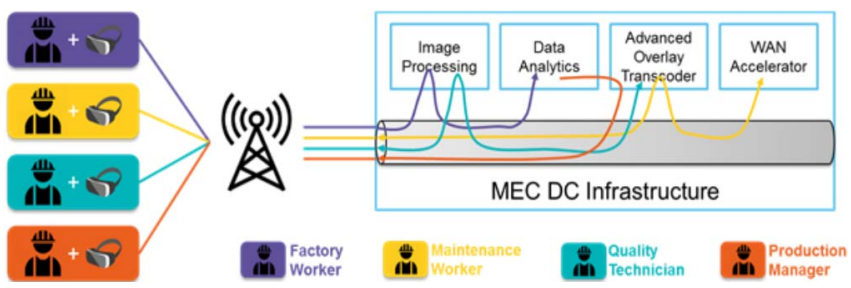

Fig. 1. Predictive Manufacturing Platform Architecture

This platform uses AR glasses to enable the input and output of information from the worker perspective. All the information sent and received by the glasses considers the context of the worker within the company. The platform itself is deployed in a DC located on the edge of the network and close to the company's premises. The traffic associated with each device is sent to the various applications according to the worker context and needs by using technologies such as dynamic service function (DSF), thus optimizing resource usage. By using this type of platforms, managers can track the different parts along the production line by using the cameras mounted on the glasses and from the information being collected from different machines and sensors. All collected data is afterwards processed by specific analytic applications which use an AI mechanism (e.g., machine learning) to predict and schedule an optimal time instante to perform predictive maintenance on the machines and related components, thus reducing downtime.

The needs to perform maintenance operation on the impacted machines is triggered by an alarm generated by the operation support system. By following this approach, the situation of machines constraining production is avoided. Further details on the Initial and Step-by-step scenarios are given in [13].

Final scenario - After the conclusion of the maintenance operation, the production line resumes normal operations and the respective notification is sent to the production manager. Analytics systems collect all the data related to the maintenance procedures, including the request for specialized assistance, so that in the future it can be used to plan and optimize the predictive maintenance, and reduce downtime.

C. Uses cases 3, 4 and 5: Jump Travelling, Multiplayer Game Using VR \& Live Events using $V R / A R$

Use cases 3, 4 and 5 are as follows, as described in [12]:

- Jump Travelling: Users at specific locations, e.g., a theme park, would like to experience a new type of roller coaster, one in which they could enjoy a jump travel, from one location to another around the world, experiencing a 360-degree view from a flying UAV, using immersive technology.

- Multiplayer Game Using virtual reality (VR): This scenario consists of an online multiplayer game where a minimum of two opponents can perform virtual battles in a virtual arena. All players must be equipped with VR devices and interaction sensors that detect their movements. Each player makes use of a virtual melee weapon to attack or defend so that all players can react accordingly.

- Live Events using VR / (AR): Users at a venue, e.g., a soccer stadium, can follow the game from different viewpoints, which can also change over time for each individual user. This is enabled by an operator places several cameras around the stadium that capture images from multiple viewpoints.

A detailed presentation of these use cases is given in [12].

\section{REQUIREMENTS}

The use cases described above will be used to provide some basic requirements to be considered in the more fundamental research. Each of them is related to one or more of the scenarios considered in 5G and include the type of communications identified in [1]. These scenarios include: enhanced Mobile Broadband (eMBB), massive Machine Type Communications (mMTC), and Ultra-Reliable and Low Latency Communications (URLLC)

The reader may find full information on requirements for the scenarios foreseen in $5 \mathrm{G}$ by the International Telecommunications Union (ITU) in the Report ITU-R M.2411-0 (11/2017), [13].

The following requirements, extracted from this report, are the most relevant for the TeamUp5G project: 
1. Peak data rate (Gbit/s): eMBB: 20 (DL), 10 (UL).

2. Peak spectral efficiency (bps/Hz): eMBB: 30 (DL), 15 (UL).

3. 5th percentile user spectral efficiency $(\mathrm{bps} / \mathrm{Hz})$ :

a) Indoor Hotspot: eMBB: 0.3 (DL), 0.21 (UL).

b) Dense Urban: eMBB 0.225 (DL), 0.15 (UL).

c) Rural: eMBB 0.12 (DL), 0.045 (UL).

4. Average Spectral Efficiency (bps/Hz/TRxP):

a) Indoor Hotspot: eMBB 9 (DL), 6.75 (UL).

b) Dense Urban: eMBB 7.8 (DL), 5.4 (UL).

c) Rural: eMBB 3.3 (DL), 1.6 (UL).

5. Area Traffic Capacity $(\mathrm{Mbps} / \mathrm{m} 2)$ : eMMB (Indoor): 10 (DL), 10 (UP).

6. User experienced data rate (Mbit/s): eMBB 100 (DL), 50 (UL).

User plane latency (ms): 4 (eMBB), 1 (URLLC).

7. Control Plane latency (ms): 20 .

8. Connection density (devices/km2): mMTC: $10^{6}$.

9. Energy efficiency: Undetailed.

10. Reliability (URLLC): the minimum requirement for the reliability is $1-10^{-5}$ success probability of transmitting a layer 2 protocol data unit (PDU) of 32 bytes within $1 \mathrm{~ms}$ in channel quality of coverage edge for the Urban macro-URLLC test environment, assuming small application data (e.g. 20 bytes' application data + protocol overhead).

11. Mobility:
a) Stationary: $0 \mathrm{~km} / \mathrm{h}$.
b) Pedestrian: $0 \mathrm{~km} / \mathrm{h}$ to $10 \mathrm{~km} / \mathrm{h}$.
c) Vehicular: $10 \mathrm{~km} / \mathrm{h}$ to $120 \mathrm{~km} / \mathrm{h}$.
d) High speed vehicular: $120 \mathrm{~km} / \mathrm{h}$ to $500 \mathrm{~km} / \mathrm{h}$.
e) Indoor Hotspot: eMBB: $1.5(\mathrm{bps} / \mathrm{Hz}), 10(\mathrm{~km} / \mathrm{h})$.
f) Dense Urban: eMBB: $1.12(\mathrm{bps} / \mathrm{Hz}), 30(\mathrm{~km} / \mathrm{h})$.
g) Rural: eMBB: $0.8(\mathrm{bps} / \mathrm{Hz}), 120(\mathrm{~km} / \mathrm{h}) ; 0.45$ (bps/Hz), $500(\mathrm{~km} / \mathrm{h})$.

12. Mobility Interruption Time: $0 \mathrm{~ms}$ (eMBB/ URLLC).

13. Bandwidth: between $100 \mathrm{MHz}$ and $1 \mathrm{GHz}(>6 \mathrm{GHz}$ ).

On figure 2 is showen the relevance of a set of requirements for the three $5 \mathrm{G}$ main scenarios.

A more detailed description of the requirements for TeamUp5G use cases is presented in the following.

\section{A. Use case 1: Emergency Drone (eMBB, mMTC)}

The foreseen requirements for this use case include those of eMBB and mMTC. Emergency Drones must be capable of transmitting video images of an extremely high quality (at least $4 \mathrm{k}$ at $30 / 60 \mathrm{fps}$ ), making it necessary to use a high broadband connection (eMMB). Besides that, it is important for the drones to be able to communicate between them, creating a network of drones and people devices (mMTC).

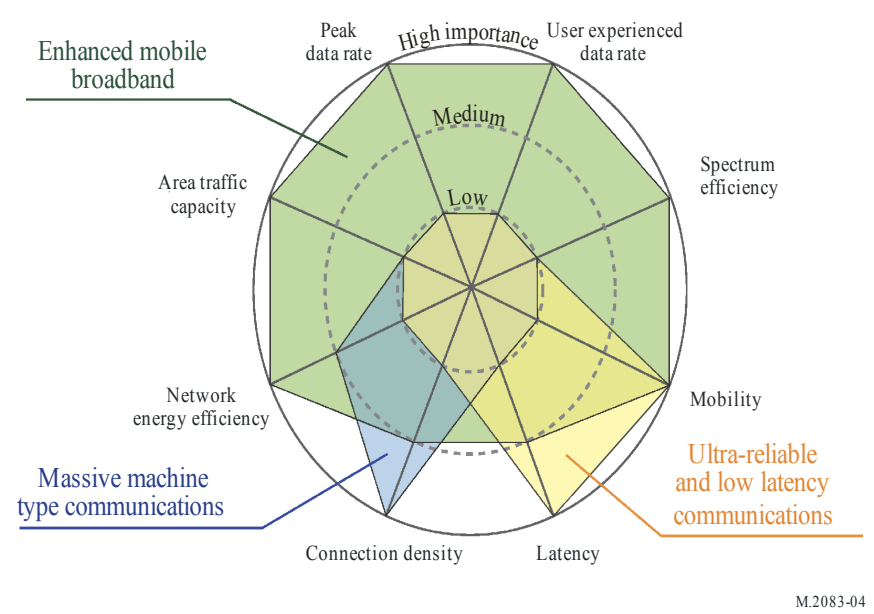

Fig. 2. Polygon describing scenarios and requirements, extracted from [11].

URLLC is not a requirement in this use case since $4 \mathrm{~ms}$ of user plane latency provided by eMBB is sufficient for this scenario.

\section{B. Use case 2: Predictive Maintenance in Manufacturing (eMBB, mMTC, URLLC)}

The foreseen requirements for this use case include those of eMBB, mMTC and URLLC.

Operators will be equipped with AR glasses to help them perform their predictions when working. This AR glasses will record, send, process in an edge cloud and respond (via some AR banner) to the operator. Thus, it is important to received meaningful information in real time (URLLC) after having sent $4 \mathrm{k}$ video (eMMB) and processed in a edge cloud. Besides this, information from sensors and machines is also sent to the central processors (mMTC).

\section{Use case 3: Jump Travelling (eMBB, URLLC)}

The foreseen requirements for this use case include those of eMBB and URLLC.

In this use case, virtual and augmented reality require very high-quality video images (eMBB) to be sent to the users enjoying "Jump Travel". Besides, the users may control drones (URLLC) that are recording and sending images "on site". mMTC is not required, since the connection/ communication with and between many devices is not expected.

\section{Use case 4: Multiplayer Game Using VR (eMBB, URLLC)}

The foreseen requirements for this use case include those of eMBB and URLLC. When playing videogames online, very low latencies may be a strict requirement to assure a proper enjoyable experience (URLLC). Besides that, since VR is expected to be used in this scenario, as stated for previous use cases, a high data rate is needed (eMBB). mMTC is not a requirement in this use case. 
Table 1: TeAmUp5G INITIAL USE CASES AND REQUIREMENTS

\begin{tabular}{|c|c|c|c|c|c|}
\hline & 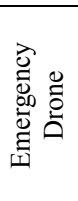 & 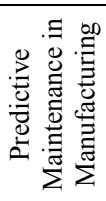 & 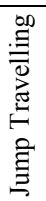 & 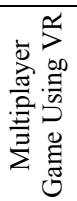 & 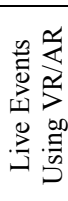 \\
\hline $\begin{array}{l}\text { Peak data } \\
\text { rate }\end{array}$ & 5 & 5 & 5 & 5 & 5 \\
\hline $\begin{array}{l}\text { User } \\
\text { experience } \\
\text { data rate }\end{array}$ & 5 & 5 & 5 & 5 & 5 \\
\hline $\begin{array}{l}\text { Spectrum } \\
\text { efficiency }\end{array}$ & 5 & 5 & 5 & 5 & 5 \\
\hline Mobility & 5 & 5 & 5 & 3 & 5 \\
\hline Latency & 3 & 5 & 5 & 5 & 5 \\
\hline $\begin{array}{l}\text { Connection } \\
\text { density }\end{array}$ & 5 & 5 & 3 & 3 & 5 \\
\hline $\begin{array}{l}\text { Energy } \\
\text { efficiency }\end{array}$ & 5 & 5 & 5 & 5 & 5 \\
\hline $\begin{array}{l}\text { Area traffic } \\
\text { capacity }\end{array}$ & 5 & 5 & 3 & 5 & 5 \\
\hline
\end{tabular}

\section{E. Use case 5: Live Events using VR/AR (eMBB, mMTC, URLLC)}

The foreseen requirements for this use case include those of eMBB, mMTC and URLLC. Since AR and VR is expected to be used in this scenario, as stated for previous use cases, a high data rate is needed (eMBB). A very low latency (URLLC) is expected to prevent a noticeable gap between reality and the images transmitted to visualization devices (VR/AR). Many users are expected in a reduced volumetric space (stadium for instance), thus capacity to communicate with several devices is needed (mMTC).

On Table $I$ it is summarized the main requirements foreseen at this first stage for the TeamUp5G use cases, that are also adressed in [1]. These will be refined while the project evolves.

\section{CONCLUSION}

This work analyses use cases we plan to implement within TeamUp5G, which will require multiple scientific and technological advancements in the different topics to be researched by the individual Early Stage Reseachers' (ESRs).

Several broad use cases have been proposed, which will be refined over the course of the next months, in order to cover (when seen as a combined set) all research areas to be pursued by the different TeamUp5G researchers. The use cases are characterized in terms of Key Performance Indicators following the recommendations from ITU. None of the proposed use cases at this moment aims to cover all topics of research, however we might consider proposing at a later stage, one or two use cases that by themselves will demonstrate the progress we will make in all areas of research covered by the project.

\section{REFERENCES}

[1] D. Corujo, C. Guimaraes, B. Santos and R. L. Aguiar, "Using an opensource IEEE 802.21 implementation for network-based localized mobility management," IEEE Communications Magazine, vol. 49, no. 9, pp. 114-123, September 2011.

[2] I. Demydov, M. Seliuchenko, M. Beshley and M. Brych, "Mobility management and vertical handover decision in an always best connected heterogeneous network," The Experience of Designing and Application of CAD Systems in Microelectronics, Lviv, 2015, pp. 103105.

[3] D. Corujo, TM Melia, AV Vidal, IS Soto, A. de la Oliva de la Oliva, R. Aguiar, "Impact of Heterogeneous Network Controlled Handovers on Multi-Mode Mobile Device Design," IEEE Wireless Communications and Networking Conf. - WCNC, Hong-Kong, China, Vol. 1, pp. 3884 - 3889, March, 2007

[4] Philipp Hock, Sebastian Benedikter, Jan Gugenheimer, Enrico Rukzio, "CarVR: Enabling In-Car Virtual Reality Entertainment", Proceedings of the 2017 CHI Conference on Human Factors in Computing Systems Pages 4034-4044

[5] www.i-scoop.eu/industry-4-

0/\#The fourth Industrial Revolution and

the third_industrial_innovation_wave_of_the_Industrial_Internet (Access: May, 2020)

[6] www.bcg.com/publications/2016/lean-manufacturing-technologydigital-sprinting-to-value-industry-40.aspx (Access: May, 2020)

[7] 5GPPP Architecture Working Group, "View on 5G Architecture" (version 2.0 Dec 2017)

[8] Eric Y. Chen, Yutong Pei, Shuo Chen, Yuan Tian, Robert Kotcher, and Patrick Tague. Oauth demystified for mobile application developers. In 2014 ACM SIGSAC, CCS '14. ACM, 2014.

[9] V. Sucasas, Georgios Mantas, A. Radwan, and J. Rodriguez. An oauth2-based protocol with strong user privacy preservation for smart city mobile e-health apps. In Communications (ICC), 2016 IEEE International Conference on, June 2016.

[10] Foteini Baldimtsi and Anna Lysyanskaya. Anonymous credentials light. In Proceedings of the 2013 ACM SIGSAC, CCS '13, pages 1087-1098, New York, NY, USA, 2013. ACM.

[11] ITU-R M.2083-0, IMT Vision - Framework and overall objectives of the future development of IMT for 2020 and beyond. ., Sep. 2015.

[12] Sérgio Figueiredo, Bruno Parreira, Diogo Costa, Luis Miguel Campos, Carlos Marques, João Pires, Jorge Martins, Luís Landeiro Ribeiro, Guilherme Santos, Jorge Sá Silva, Fernando Boavida, Pedro Ferreira, Ngombo Armando, Duarte Raposo, André Braga Reis, Bruno Areias, Nuno Humberto Paula, Susana Sargento, Daniel Corujo, Rui Silva, David Santos, Valdemar Monteiro, Georgios Mantas, Victor Sucasas and Jonathan Rodriguez, "Scenario definition for different traffic profiles ," MOBILIZADOR 5G - Components and services for 5G networks (Project No. 24539), Altice labs, Aveiro, Portugal, June 2018.

[13] ITU-R M.2410-0 (11/2017), Minimum requirements related to technical performancefor IMT-2020 radiointerface(s). https://www.itu.int/dms_pub/itu-r/opb/rep/R-REP-M.2410-2017PDF-E.pdf

[14] www.cleverism.com/industry-4-0/ (Access: May, 2020) 